\title{
ANTECEDENTES DE ALGUNOS LINEAMIENTOS DE LA ESTRATEGIA DE ARISTÓTELES FRENTE AL NEGADOR DEL PRINCIPIO DE NO CONTRADICCIÓN EN EL SOFISTA DE PLATÓN
}

\begin{abstract}
The purpose of this paper is to show that in the Sophist Plato develops a dialectical strategy that appeals to necessary conditions of language as a fundamental step toward establishing some principles of his ontology. This strategy constitutes a clear antecedent of the elenctic refutation offered by Aristotle in Metaphysics Gamma 4 against the denier of the principle of noncontradiction and could be described as transcendental because it refers to conditions without which there wouldn't be any speech. The paper aims to show that such a strategy is used both by Plato and Aristotle to deal with radical adversaries whose refutation allows the establishment of basic theses of their own philosophies.
\end{abstract}

Keywords

Plato - Aristotle - dialectics - refutation- transcendental arguments

A lo largo de los últimos años algunos autores han señalado ciertos paralelismos entre la estrategia dialéctica utilizada por Aristóteles frente al negador del principio de no contradicción (PNC) en Metafísica Gamma 4 y aquella utilizada por Platón contra las posiciones relativistas. ${ }^{1}$ En efecto, se ha ofrecido una serie de lecturas que apunta fundamentalmente al célebre argumento de la auto-refutación presentado en Teeteto como antecedente directo de ciertos elementos de la prueba aristotélica. Sin embargo, de una indagación en los diálogos platónicos tardíos y en especial del Sofista, emergen otros puntos de contacto escasamente estudiados. ${ }^{2}$ Buscaré entonces emprender esta indagación focalizándome en las estrategias utilizadas en algunos argumentos medulares de ambos textos. Pretendo exhibir que, en sus grandes trazos, ciertas estrategias o argumentos del Sofista que involucran el recurso a la posibilidad misma del lenguaje constituyen un antecedente claro de la primera prueba aristotélica ofrecida en Metafísica Gamma 4 en favor del principio de no contradicción (PNC). Apunto a mostrar que, al igual que en el caso de Aristóteles, tal recurso de carácter dialéctico es utilizado por Platón a la hora de enfrentarse a adversarios radicales cuya refutación permite asentar tesis fundantes de la propia filosofía.

Para exhibir los puntos de contacto mencionados esbozaré, en primer lugar, en sus grandes líneas el argumento ofrecido por Aristóteles contra el negador del PNC. Sin ningún afán de profundizar en este complejo y polémico argumento, me interesa en particular exhibir

\footnotetext{
${ }^{1}$ Al respecto cabe destacar los trabajos de P. GotTLIEB, The Principle of Non-Contradiction and Protagoras: The Strategy of Aristotle's Metaphysics IV 4, in Proceedings of the Boston Area Colloquium in Ancient Philosophy, Vol. VIII (1994), pp. 183-209 y a M. NARCY, Platon revu et corrigé, in B. CASSIN y M. NARCY, La décision du sens. Le livre Gamma de la Métaphysique d'Aristote, Paris 1984.

${ }^{2}$ En el notable artículo de J. H. KÖNIGSHAUSEN, ¿Paralelismos entre el Sofista de Platón y el libro Gamma de la Metafísica de Aristóteles?, en Anales del Seminario de Historia de la Filosofía, № 10 , Madrid 1993, pp. 157-172, el autor, justificando la interrogación que constituye el título de su artículo señala: "en vista de la inmensa literatura dedicada a los escritos platónicos y aristotélicos, apenas parece creíble que tales paralelismos, si los hubiera, no hubiesen sido ya puestos de relieve desde hace mucho.". Como excepción es posible señalar el artículo de D. C. BALTZLY, To an Unhypothetical First Principle in Plató 's Republic, «History of Philosophy Quarterly», XIII (2) (1996) pp. 149-165, que indaga en los puntos de contacto entre las estrategias argumentativas específicamente dialécticas en la obra platónica y la prueba a favor del principio de no contradicción en Aristóteles.
} 
sus aristas trascendentales. Aspiro a mostrar que el argumento se deja encuadrar en un tipo de estrategia que supone acudir a las condiciones de posibilidad de todo discurso posible.

En segundo lugar, me referiré muy esquemáticamente a cuatro argumentos medulares ofrecidos en el Sofista contra diversas tesis: los esgrimidos contra quien sostiene la posibilidad de lo falso, contra el pluralista, contra el monista y contra quien niega la posibilidad de la combinación tanto al nivel del enunciado como a nivel ontológico. En todos ellos, me propongo mostrar, se pone en juego un dispositivo argumentativo que llamaremos "radical", en la medida que recurre a las condiciones de posibilidad de la formulación de la propia tesis por parte del adversario.

Para poder referirme a los puntos de contacto entre el Sofista y el primero de los argumentos de Metafisica Gamma 4 voy a bosquejar la estructura general del polémico argumento de Aristóteles contra el negador del principio de no contradicción (PNC). Ante todo, interesa enmarcar la estrategia argumentativa utilizada por el estagirita en el horizonte de los argumentos trascendentales. ${ }^{3} \mathrm{Si}$ bien en la filosofía kantiana "trascendental" refiere a nuestros conceptos a priori de los objetos (Crítica de la razón pura A 11-12/B25), en la discusión actual este término refiere a una clase particular de argumento cuya naturaleza es objeto de polémica en la medida que su forma no se distingue de la de un argumento deductivo. ${ }^{4}$ Sin embargo, se suele destacar como su nota distintiva el hecho de que plantea que algo es condición necesaria de posibilidad de otra cosa, de modo tal que esta última no podría darse sin la primera. Es importante establecer igualmente que tal tipo de argumento constituye una herramienta dialéctica en la medida que busca concluir condiciones de posibilidad de un cierto de tipo de experiencia, de conocimiento o de lenguaje a partir de la emisión de la tesis por parte del adversario, el cual sostiene usualmente posiciones escépticas o relativistas. Así, pretende mostrar que para que se den ciertas instancias cuya existencia el interlocutor admite (y no puede rechazar en la medida que quiera sostener su discurso), es necesaria a su vez la existencia de principios de los cuales es imposible dudar sin cuestionar el propio punto de partida. ${ }^{5}$ Puesto que consiste en la identificación de condiciones de posibilidad, el argumento trascendental consta básicamente de dos pasos: en primer lugar, se le confiere la palabra al adversario o se afirma algo que constituya un punto de partida incuestionable para él; ${ }^{6} \mathrm{y}$, en segundo lugar, se demuestra que, de no darse cierta condición o conjunto de condiciones, tal afirmación no sería posible. De suerte que se concluye la necesidad de tal condición o conjunto de condiciones. ${ }^{7}$

\footnotetext{
${ }^{3}$ El carácter trascendental de la prueba aristotélica y del principio aristotélico ya ha sido establecido entre otros por E. BERTI, Studi aristotelici, L'Aquila 1975, p. 83; R. M. DANCY, Sense and Contradiction: A Study in Aristotle, Dordrecht 1975, B. CASsin, La Décision du Sens, Paris 1989 y B. Hafemann, Aristoteles' Transzendentaler Realismus. Inhaltund Umgangerster Prinzipien in der 'Metaphysik', Berlin - N. York 1998.

${ }^{4}$ Cfr. M. GRAM, Must Transcendental Arguments be Spurious, en «Kant Studien» LXV (1974) pp. 304317 , p. 304. A la hora de definirlos se suele tener en vista una serie de argumentos filosóficos de diversas épocas y corrientes. Para acceder a algunas de sus caracterizaciones clásicas cfr. B. STROUD, The Goal of Transcendental Arguments, in R. STERN (ed.), Transcendental Arguments. Problems and Prospects. Oxford 2003 p. 158; STERn, op, cit. p. 2 ss.; I. CABRERA(ed.) Argumentos trascendentales. México D. F. 1999, p. 7 y ss. y R. S. WALKER, Argumentos trascendentales y escepticismo, en I. CABRERA (ed.), op.cit., pp. 223-251, pp. 240.

${ }^{5}$ B. STROUd señala que sería mejor hablar de "estrategias trascendentales" asumiendo así el pleno carácter dialéctico y refutativo que caracteriza a tales argumentos (op.cit., pp. 157-160).

${ }^{6}$ Es importante destacar que el carácter de irrechazable de tal punto de partida sería, de acuerdo con algunas lecturas, lo que caracteriza en última instancia tal tipo de argumentación. De modo que lo que le conferiría una particular tonalidad frente a otros argumentos de carácter demostrativo sería la fuerza dialéctica del punto de partida.

${ }^{7}$ Eludo aquí el polémico punto acerca de la forma lógica que asumen tales argumentos. Algunos han defendido que se trata de una prueba indirecta de reductio ad absurdum para probar la necesariedad que liga la condición a demostrar y el punto de partida incuestionable. KANT, sin embargo, rechaza para las demostraciones trascendentales tal tipo de pruebas indirectas y exige que sean de carácter "ostensivo", es
} 
En Metafísica Gamma 4 Aristóteles se propone una especie de prueba del primero y más firme de los principios, el principio de no contradicción (PNC). Establece, sin embargo, la imposibilidad de una demostración, ya que al hacerlo se incurriría en petición de principio en la medida que el PNC está supuesto en toda demostración. Es posible ofrecer, sin embargo, un particular tipo de prueba:

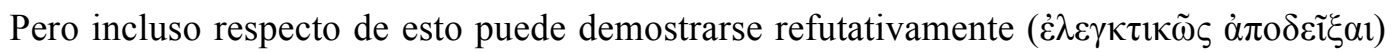

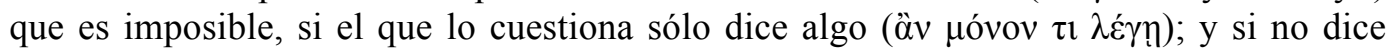
nada, sería ridículo buscar un discurso frente a quien no está dotado de un discurso de nada, en tanto no lo está. Pues tal persona en tanto tal es similar a una planta. Digo que "demostrar refutativamente" difiere también de "demostrar", porque al demostrar parecería que uno comete petición de principio, pero al ser otro responsable [de cometerla], sería refutación y no demostración. $(1006 a 11-16)^{8}$

De modo que si bien no es posible una demostración estricta del PNC por el hecho de constituir un principio, sí cabe una especie de refutación de quien pretenda negar su validez. Esta consiste en una prueba dialéctica, pues parte de lo dicho por el adversario. Esto se condice perfectamente con lo establecido por Aristóteles en Tópicos I 2 101a, texto en que se establece como una de las utilidades de la dialéctica justamente la posibilidad de discurrir sobre "las cosas primeras ( $\tau \grave{\alpha}$ $\pi \rho \tilde{\omega} \tau \alpha)$ de cada una de las ciencias": puesto que es imposible decir algo acerca de los principios propios de la ciencia a partir de ellos mismos, es necesario discurrir sobre ellos mediante las éndoxa con ellos relacionadas. ${ }^{9}$ En el caso de Metafísica Gamma 4 nos ubicamos, pues, en un escenario claramente dialéctico en la medida que aquello que estamos intentando probar es justamente el primero de los principios. El hecho de suponer la necesidad de un adversario, por un lado, y el de asumir como punto de partida el discurso del adversario, por el otro, constituyen las primeras condiciones dialécticas del argumento, que se condicen con condiciones trascendentales.

Aristóteles subraya que el punto de partida no consiste en pedirle al adversario que diga que algo es o no es, en cuyo caso se cometería petición de principio, sino en pedirle "que diga

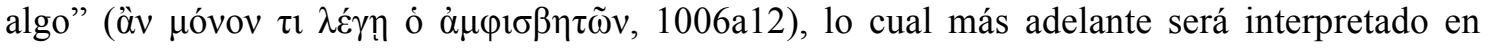
términos de significar algo para sí mismo y para otro ( 1006a21). Si alguien concede eso, habrá demostración, "pues ya habrá algo determinado" ( $\tau 1$

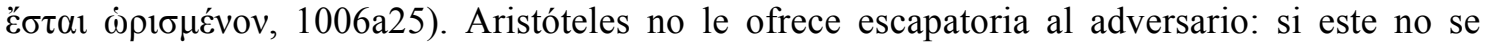
sujeta a la condición de significar algo, no dice nada (1006a22-24). En tal caso, afirma, se convertiría en una planta en la medida que no ejerce aquella capacidad específica del hombre. Es en este punto que uno puede sospechar que Aristóteles recurre al límite que impone el escenario dialéctico: quien no habla se convierte en una nada dialéctica, no puede erigirse en

decir que conecten las verdades que se quiere demostrar con sus condiciones de posibilidad (Crítica de la razón pura A 789-B817-822). Sin embargo, algunos comentaristas consideran que el mismo KANT utiliza un modo apagógico o indirecto (M. GRAM, Transcendental Arguments, «Nous» V (1971) pp. 20-26) y que, en última instancia, todo argumento trascendental debería poder traducirse a una forma indirecta. Es interesante señalar que también respecto del argumento que aquí nos interesa de Aristóteles, aquel dirigido contra el negador del principio de no contradicción, encontramos una disputa similar acerca de su forma. Al respecto ver infra $\mathrm{n}$. 14.

${ }^{8}$ Las traducciones ofrecidas son propias. Sigo aquí las ediciones de Oxford tanto para las citas de Platón como para las de Aristóteles. (Platonis Opera, recognoverunt brevique adnotatione critica instruxerunt E. A. Duke -W. F. Hicken-W. S. Nicoll- D. B. Robinson e J. C. G. Strachan, t. I, Oxford University Press, Oxford 1995 y Aristotelis Metaphysica, recognovit brevique adnotatione critica instruxit W. JAEGER, Oxford University Press, Oxford 1957). Agradezco muy especialmente a Marcelo Boeri, cuyas acertadas sugerencias me ayudaron a precisar las traducciones.

${ }^{9}$ Al respecto cfr. Anal. Pr. II, 2066 b 11-12. Según establece R. M. DANCY, op. cit., p. 14 la clave para distinguir la demostración de la refutación se encuentra justamente en su dimensión dialéctica: una refutación supone siempre el discurso de un adversario. Cfr. también Metaph. K 5 1062a2-3. Dejamos de lado aquí el espinoso problema del carácter que asumen las éndoxa en el contexto de la dialéctica "fuerte" o "radical" ejercida por Aristóteles en este contexto. 
adversario al no poder emitir discurso alguno. Recurre así a un escenario que podríamos considerar previo al de la enunciación de la propia tesis por parte del adversario, puesto que lo que se pone en juego, en última instancia, es la posibilidad misma de hablar, la cual comporta la posibilidad de ser considerado hombre. En efecto, afirma Aristóteles "si no [significara nada] entonces no habría discurso ( $\lambda$ ó $\gamma$ os) para tal persona, ni con relación a sí mismo ni con relación a otro" (1006a22-24). Acorralado, el adversario se vería en la obligación de decir algo, y si lo hace, sostiene Aristóteles, ya nos encontramos frente a un discurso significativo, lo cual supone algo determinado y, en la medida que la determinación comporta el PNC, ya se habrá demostrado la validez del principio. ${ }^{10}$ Sin embargo, señala, el responsable de tal demostración

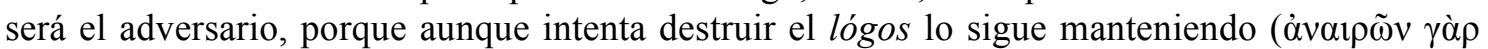

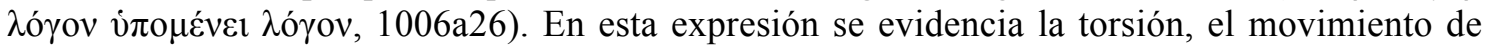
auto-bloqueo que, de acuerdo con Aristóteles, realiza el adversario a través del propio acto de habla. Se verifica entonces el modo en que la carga de la prueba es invertida al hacer responsable al adversario de servirse del principio que él pretende rechazar y Aristóteles demostrar. De aquí se infiere la remisión a una cadena de condiciones: el lógos (cualquiera sea) implica la significación, esta nos reenvía a la determinación como precondición y, a su vez, la determinación nos reenvía al PNC, de suerte que de lo que se trata es de recurrir a los prerrequisitos de todo discurso.

A partir del panorama descripto es posible apreciar la prueba en toda su radicalidad: así como desde el punto de vista de la tesis a refutar Aristóteles se retrotrae de la posibilidad del discurso del adversario a la de todo discurso, desde el punto de vista de quien sostiene la negación del PNC, se retrotrae de la posibilidad de considerarlo en tanto adversario a la de considerarlo en tanto hombre. En definitiva, quien no se sujete al PNC no puede hablar y, en consecuencia, no puede ser considerado partícipe del género humano.

Es muy importante hacer hincapié en el hecho de que la prueba aquí ofrecida modifica dos rasgos definitorios de las refutaciones, las cuales en primer lugar, parten de enunciados concedidos por el oponente; y, en segundo lugar, tienen por objetivo deducir la contradictoria de la tesis por él sostenida. ${ }^{11}$ En el caso particular de esta prueba, según aclara Aristóteles en el controvertido pasaje 1006a29-31, no se parte de un enunciado sino de una palabra (ővo $\alpha$ ) emitida por el adversario, de un término significativo. La exigencia que se le formula al

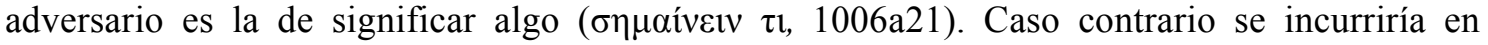
petición de principio, pues el adversario se vería obligado a decir que algo es o que algo no es para contar con una premisa a partir de la cual efectuar una refutación, lo cual supone pedirle que acepte el principio. Podemos sospechar que el adversario podrá negarse afirmando que algo es y no es. ${ }^{12}$ En segundo lugar, Aristóteles no probará la contradictoria de la tesis del oponente a partir de una deducción, sino que exhibirá la condición supuesta a partir de la cual tal

\footnotetext{
${ }^{10}$ Estoy eludiendo acá múltiples problemas envueltos en la prueba, el primero de los cuales reside en determinar si esta apunta a establecer la necesaria creencia en el PNC por parte del interlocutor o su validez objetiva (o si a través de la primera se intenta alcanzar la segunda). Aun sin expedirnos sobre esta compleja cuestión, podemos mostrar nuestro punto: que Aristóteles se sirve de la argumentación trascendental para esta demostración del PNC (cualquiera sea el nivel que esté en juego).

${ }^{11}$ Aristóteles debe introducir algún cambio en la estructura de la refutación, pues en Anal. Pr. II, 20 66b11 el élenchos es definido como "un silogismo que deduce la contradictoria de una tesis dada". Así, puesto que éste constituye un silogismo, ya presupone la aceptación de la validez del PNC. Acerca de si la argumentación aristotélica a favor del PNC constituye una reductio ad absurdum, en cuyo caso su estructura sería deductiva y presupondría el principio en cuestión o no, véase J. LUKASIEWICZ, On the principle of contradiction in Aristotle (trad. V. Wedin), «Review of Metaphysics» XXIV (1971) pp. 485509, p. 508; B. CASSIN, op. cit. pp. 17-25; R. M. DANCY, op. cit., pp. 19-20. Si efectivamente el argumento escapa a esta estructura, nos encontraríamos frente a un rasgo importante que lo aproxima, según la caracterización trazada, a los argumentos trascendentales.

${ }^{12}$ Reconstruyo aquí el sentido de la prueba sobre la base de una interpretación del texto según la cual en el pasaje 1006a19-21 Aristóteles no le solicita al interlocutor que diga que algo es o no es, lo cual supondría, como él mismo aclara, cometer petición de principio, sino sobre la base de la lectura que entiende que el requerimiento de Aristóteles es que signifique algo con sentido para sí mismo y para el otro. Que se trata de un término significativo aislado se sigue del ejemplo del que se valdrá Aristóteles, "hombre". De suerte que uno de los nudos más controversiales del texto lo interpretamos en ese sentido.
} 
enunciación se puede llevar a cabo. Según hemos establecido previamente, este constituye un segundo rasgo definitorio de las argumentaciones trascendentales: no se pretende extraer conclusiones a partir de las premisas sino reconducir a sus mismas condiciones.

Algunos intérpretes han subrayado el hecho de que habría comprometida aquí una "oposición pragmática" entre el acto lingüístico realizado y el significado de la afirmación hecha, de suerte que en el mismo acto de enunciar la propia tesis se evidenciaría una contradicción: si el PNC es una condición de la significatividad de todo discurso, afirmar con sentido "el PNC es falso" presupone que ya se ha aceptado la validez del PNC. ${ }^{13} \mathrm{La}$ contradicción pragmática apunta a la posición misma en la cual la refutación pone al adversario: si bien este rechaza el principio, acepta sin embargo sostener su discurso, es decir, defender su tesis contra la contradictoria. Sería entonces su actitud la que supondría una auto-refutación. Por mi parte, entiendo que si bien esta representa una dimensión importante de la prueba, sin embargo no agota su alcance. En primer lugar, cabría aclarar que si bien lo que pone al adversario en el lugar dialéctico en que se ubica es su cuestionamiento del PNC, tal tesis no es el punto de partida asumido por Aristóteles. ${ }^{14}$ En segundo lugar, y en estrecha relación con lo señalado recién, el argumento se pretende más radical que la prueba pragmática, pues para ejercer la refutación ni siquiera es necesaria la formulación de la tesis del adversario. Alcanza con que el adversario diga una palabra con sentido. A lo que se apunta, pues, es a la condición de cualquier discurso y no específicamente a la de aquel emitido por el adversario. Aristóteles se asienta así, según hemos sostenido, en un territorio previo al de la tesis a refutar. Es por eso que el marco que le confiere más inteligibilidad al argumento es el de los argumentos trascendentales. ${ }^{15}$

Así, más allá de la controversia en torno a cuáles podrían ser los requerimientos iniciales de Aristóteles con respecto al interlocutor y a qué sea lo implicado en el decir algo requerido, es importante destacar que la prueba procede frente a un adversario e indaga en las condiciones de posibilidad de cualquier enunciación posible. De modo que, como el mismo Aristóteles señala, tal tipo de prueba apunta a constreñir al adversario a admitir la tesis del PNC en la medida en que quiera hablar, ${ }^{16}$ requerimiento que no podrá rechazar en la medida que quiera seguir perteneciendo al género humano. ${ }^{17}$

\footnotetext{
${ }^{13} \mathrm{~A}$. CASSINI, La justificación aristotélica del principio de no contradicción, tesis doctoral inédita, Universidad de Buenos Aires, 1990, p. 167. Cfr. también R. M. DANCY, op.cit., p. 19.

${ }^{14}$ Tal condición es subrayada por L. CASTAGNOLI, Ancient Self-Refutation. The Logic and History of the Self-Refutation Argument from Democritus to Augustine, Cambridge University Press, Cambridge, 2010, pp. 88-94, quien considera que la prueba de Aristóteles no constituye una auto-refutación en la medida que el punto de partida explícitamente establecido por Aristóteles no es la tesis del adversario sino la emisión de una palabra significativa para sí mismo y para el otro (1006a21). CASTAGNOLI asume, como propone M. V. WediN en Some logical problems in Metaphysics $\Gamma$, «Oxford Studies in Ancient Philosophy» XIX (2000) pp. 113-62, at p. 129, que la estructura de la prueba es directa y procedería del siguiente modo: 's' significa (establecido por el interlocutor); 's' significa implica el PNC (establecido por Aristóteles y admitido por el adversario); por lo tanto, PNC. La negación del principio no figura, pues, entre las premisas de su propia refutación, o sea que la tesis a rebatir está ausente en la prueba. CASTAGNOLI señala sin embargo, que la prueba es de naturaleza dialéctica: aun cuando el punto de partida no es la tesis del adversario, sí es una palabra enunciada por él.

${ }^{15}$ Cfr. B. CASSIN, op.cit., pp. 24-5 y E. BERTI, op. cit., p. 83.

${ }^{16}$ Con respecto a la pretensión constrictiva de este tipo de argumento cfr. Metaph. 1009a15-22 en que Aristóteles mismo establece una diferencia entre los adversarios a los que se debe persuadir y aquellos a

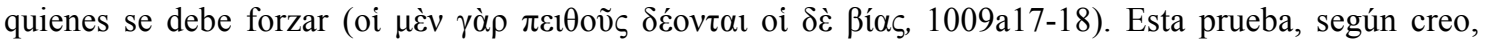
apunta justamente a estos últimos, cuya curación consistirá en oponerles una refutación "de su discurso en

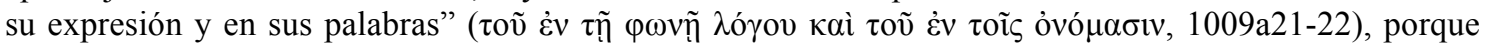

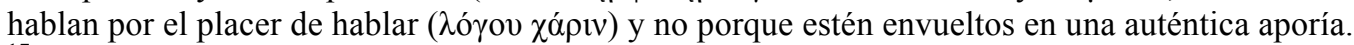

${ }^{17}$ Como hemos señalado siguiendo a CASTAGNOLI, lo que está en juego acá no es tanto una negación del discurso particular emitido por el adversario, sino la imposibilidad de cualquier discurso. Cabe destacar, sin embargo, que el adversario es tal por haber negado previamente la validez del PNC. El rol dialéctico ya está prefijado en el texto de Aristóteles cuando presenta a sus adversarios en los siguientes términos de "algunos piden una demostración del principio por ignorancia" (1006a5-6). De modo que aun cuando la prueba no se sirva de la negación del PNC como premisa, esta sí se encuentra en el horizonte inmediato.
} 
Luego de la esquemática presentación del primer argumento de Aristóteles contra el negador del PNC, en este segundo apartado apunto a mostrar que también en el Sofista se ofrece una serie de argumentos centrales que parten del factum del lenguaje y se retrotraen a sus condiciones de posibilidad, para refutar a un adversario que mantiene una tesis que pone en cuestión principios fundamentales de la ciencia que se aspira a desarrollar en este diálogo. Tales argumentos pretenden forzar al oponente a reconocer como válida la tesis del filósofo en la medida que quiera formular la propia. ${ }^{18}$ De modo que, como la prueba aristotélica, ellos no apuntan a rechazar el contenido de la tesis en cuestión acudiendo a lo que de ella se sigue, sino poniendo en juego la misma posibilidad del lógos. Así, interesa mostrar que si es posible enmarcar la prueba aristotélica contra el negador del PNC en tanto que argumento trascendental, también algunos argumentos del Sofista pueden ser leídos, al menos parcialmente, a partir de idéntico marco conceptual. Esto involucra una determinada estrategia dialéctica que podemos interpretar en tanto que refutación radical, pues aspira a poner en jaque al adversario -también él radical en la medida que niega principios básicos y manifiestos $-{ }^{19}$ acudiendo a principios anteriores a la enunciación de su propia tesis. Interesa mostrar, por otro lado, que las pruebas en cuestión se enmarcan en una estrategia general del diálogo que consistiría en postular ciertos principios de orden ontológico a partir de una indagación que parte del factum del lenguaje y se retrotrae a sus condiciones. En este sentido, los argumentos que mencionaré no constituyen meras autorefutaciones, sino que además se retrotraen a las condiciones de posibilidad de todo discurso y, en algunos casos, permiten establecer principios que se erigirán en pilares ontológicos. ${ }^{20}$ Así, aun cuando el estilo de la refutación se aleja claramente del formato socrático y cuando la dialéctica en algunos pasajes parece asimilada al método de división y reunión, hemos de ver que en el Sofista se verifica una compleja dialéctica que retoma el carácter refutativo de los primeros diálogos y lo radicaliza en la medida en que no procede echando luz sobre aquello que se sigue de la tesis del adversario, sino de sus condiciones de enunciación. ${ }^{21}$ Este movimiento

\footnotetext{
${ }^{18}$ En este sentido, presentan una estructura auto-refutativa mucho más clara que la prueba aristotélica. Con respecto a los antecedentes de este tipo de abordajes con relación a los argumentos platónicos cfr. R. Wilmet, Platonic Forms and the Possibility of Language, «Revue de Philosophie Ancienne» VIII (1990) 1 pp. 97-118 y CASTAGNOLI, op. cit. pp. 218-248.

${ }^{19}$ En este punto también podríamos encontrar una paralelismo con el texto de Metaph. Gamma que alude justamente al negador del PNC como una suerte de necio que, por ignorancia, pretende una demostración de todo (1006a5-6). Con respecto a las figuras históricas en cada caso encubiertas en el diálogo bajo la máscara del adversario, son muchas las hipótesis en juego. De todos modos, el rol funcional que asumen tales figuras en la arquitectura del diálogo permite sospechar que no necesariamente refiere a tesis defendidas en los términos que allí se presentan.

${ }^{20}$ En "Do Transcendental Arguments have a Future?” (en «Neue Hefte für Philosophie», XIV (1978) pp. 23-56) M. GRAM cuestiona la caracterización de trascendental que le confiere T. IRWIN (Aristotle's First Principles, Oxford 1990) al argumento de Aristóteles en favor del PNC y lo hace señalando que, sobre la base de la reconstrucción por él realizada de tal argumento, lo único que hace es mostrar la autocontradicción supuesta en la posición del adversario, y no que la negación de tal proposición sea una condición necesaria de nada excepto de su propia verdad. Es decir que de acuerdo con la lectura que hace GRAM de la reconstrucción de IRWIN, aquello que se establece en tanto que condición no es de índole trascendental. Entiendo que la consideración de GRAM puede ser adecuada a la luz de la reconstrucción de la prueba de IRWIN. Pero tal reconstrucción no resulta la más apropiada desde el momento que asume que el punto de partida del argumento es una afirmación del tipo "S es y no es p", lo cual supone desatender el texto de Aristóteles. A partir de la reconstrucción aquí bosquejada considero, en cambio, que las objeciones de GRAM para considerar trascendental la prueba en cuestión no se mantienen en pie.

${ }^{21}$ Muchas lecturas han entendido que aun cuando el método asume una importancia decisiva en el período tardío (en especial en Sofista y Político), Platón no propiciaría tal identificación. Al respecto cfr. J. PHILIP, Platonic Diairesis, in «Transactions and Proceedings of the American Philological Association» XCVII (1966) pp. 348-50, J. TREVASKIS, Division and Its Relation to Dialectic and Ontology in Plato, «Phronesis» XII, N², (1967) pp. 118-129, p. 120, G. RYLE, Plato’ Progress,
} 
dialéctico que rige las indagaciones ontológicas del Sofista echa especial luz sobre la polémica afirmación de 259e: "La aniquilación más completa de todo discurso consiste en separar cada cosa de las demás, pues el lógos se ha generado para nosotros, por la combinación mutua de las formas”. Al margen de las múltiples lecturas que admite este importante pasaje, es posible encontrar cristalizado en él el mecanismo general del diálogo que supone partir del discurso para desde allí reconducir a los principios. La afirmación citada vendría entonces a sellar este movimiento dialéctico que, mediante diversas formas, va del discurso hacia sus condiciones. ${ }^{22}$

Mencionaré, para apoyar esta idea, tan solo tres refutaciones medulares ofrecidas en el diálogo: la refutación del pluralista, el argumento esgrimido contra el monista y el formulado contra quien niega la posibilidad de la predicación. ${ }^{23}$ Sin embargo, antes de sobrevolar tales argumentos, quisiera apuntar que esta estrategia puede ser considerada un arma robada al adversario. En este sentido, resulta necesario referir a un argumento previo a aquellos que adelantaría la matriz auto-refutativa y, en algunos casos, trascendental, que se pondrá en juego en los otros tres. Desde un comienzo el sofista se refugia en el lenguaje al sostener que decir lo que no es es no decir nada. En efecto, el Extranjero de Elea afirma que caracterizar el sofista

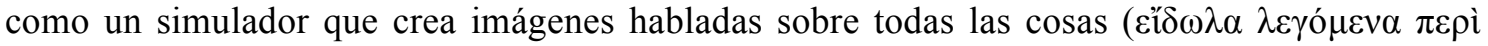
$\pi \alpha ́ v \tau \omega v, 234 c)$ introduce a quien lo afirma en una aporía, en la medida que "decir y juzgar que lo falso es realmente y enunciar $(\varphi \theta \varepsilon \gamma \xi \dot{\alpha} \mu \varepsilon v o v)$ esto sin caer en una contradicción es terriblemente difícil" (236d-e). Más allá de las diversas interpretaciones que admite este pasaje, sí se pone de relieve enseguida que lo que conduce a una contradicción es enunciar ( $\varphi \theta \varepsilon ́ \gamma \gamma \varepsilon \sigma \theta \alpha 1,237 \mathrm{a} 1)$ que hay falsedad. ${ }^{24}$ La contradicción a la que se alude no surge de confrontar el enunciado en cuestión con algún otro: es el hecho de afirmar tal cosa lo que supone incurrir ipso facto en contradicción. En efecto, el sofista argumenta en favor de la imposibilidad de decir falsedad alegando que el empleo del lenguaje nos compromete necesariamente con algo ( $\tau 1,237 \mathrm{~d})$, lo cual supone al menos la unidad y en general, el número, $\mathrm{y}$ este no puede sino agregarse a lo que es. Así, su posición supone la postulación de una "transitividad ontológica" en la medida que establece que todo decir es decir algo y este algo necesariamente es algo que es, de suerte que todo lógos es verdadero y no es posible decir lo que no es. ${ }^{25}$ Es por eso que afirmar que lo falso es supone conferirle ser al no ser, e incurrir así

Cambridge 1966, pp. 139-141, S. Rosen, Plato's Sophist. The Drama of Original and Image, New Haven 1983, p. 259 y L. Álvarez, La función propedéutica del sofista y la emergencia del filósofo. División, dialéctica y paradigmas en el diálogo Sofista, «Areté», XXIX (2016) (en prensa).

${ }^{22}$ Este movimiento, según veremos, supone en algunos casos acudir al lógos específico del interlocutor, para exhibir, por ejemplo la combinación en él implicada y, en otros casos, simplemente el hecho de que el interlocutor emita un discurso. En algunos casos, entonces, es el acto mismo de la enunciación lo que supone incurrir en contradicción, en otros es lo que se dice (en su estructura) lo que supone negar la propia tesis.

${ }^{23}$ No son, sin embargo, los únicos argumentos radicales desplegados en el diálogo. Tal como establece y analiza J. L. CASTAGNOLI, op. cit. p. 223-224, es posible considerar que el argumento desplegado conjuntamente en contra de los amigos de las formas y contra quienes afirman el movilismo universal en 248a-249c constituye también una auto-refutación: "[Valiéndonos de cualquier argumento] debemos luchar contra aquel que, eliminando el conocimiento, el pensamiento y el intelecto, mantiene obstinadamente (i $\sigma \chi v \rho i \zeta \eta \tau \alpha \iota)$ cualquier cosa acerca de algo" (249c). No abordaré aquí tal argumento porque me interesa focalizar en aquellos que remiten a las condiciones que impone el lenguaje y no el pensamiento, dimensión de la que parten tales argumentos y que supone un mecanismo "menos inmediato", por decirlo de alguna manera.

${ }^{24}$ Con relación al término $\varphi \theta \varepsilon \dot{\gamma} \gamma \varepsilon \sigma \theta \alpha \mathrm{r}$ cfr. P. CRIVELli, Plato's Account of Falsehood, A Study of the Sophist, Cambridge, 2012, pp. 34-35 quien luego de ofrecer un análisis semántico del término, pasible de traducirse como "pronunciar" o "enunciar", se pregunta por qué Platón lo utiliza en el contexto del primer argumento aporético sobre el no ser en $237 \mathrm{~b}$. Una posible respuesta es que es el hecho de la enunciación lo que Platón pretende poner de relieve a través del uso de este término: la misma pronunciación de las expresiones "no ser" y "falso" nos pone en aprietos.

${ }^{25}$ En rigor, este no ser absoluto al que aquí se alude no puede ser objeto ni de discurso ni de pensamiento (cfr. 238c). Es esta negativa rotunda la que da lugar a la consideración de un no ser relativo que sí podrá combinarse con lo que es. Al respecto cfr. la ecuación que Proclo le atribuye a Antístenes en su 
en contradicción. En razón de tales dificultades el Extranjero afirmará que el sofista se ha escondido en un terreno muy difícil (239c): las condiciones mismas que impone el discurso -el hecho de referirnos por necesidad a un algo (que es)- suponen la negación de la tesis de aquel que pretende demostrar que es posible la falsedad, es decir, el hecho de decir lo que no es.

En 238d, retrotrayéndose del problema de la falsedad al del no ser en aquel implicado, el Extranjero afirma:

¿No piensas, sobre la base de las cosas dichas, que el no ser conduce a una aporía (cic

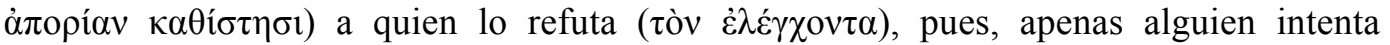

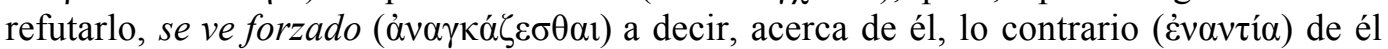
mismo? (238d)

Con relación a este pasaje, quisiera destacar un par de elementos que se verifican también en el mencionado argumento de Aristóteles contra el negador del PNC. En primer lugar, el hecho de que tal tipo de argumentación supone una suerte de inversión de la carga de la prueba (pues la tesis con que se intentaba refutar termina siendo objeto de refutación); y, en segundo lugar, la pretensión de forzar al adversario a admitir la tesis que intenta refutar. ${ }^{26} \mathrm{Me}$ interesa mostrar entonces que esta matriz de argumentación utilizada por el sofista para negar la posibilidad de lo falso refugiándose en las condiciones que impone el discurso será asumida por el extranjero a lo largo del diálogo para enfrentar a diversos adversarios, razón por la cual la estrategia general asumida parece un arma robada al enemigo. Antes de seguir adelante es necesario aclarar que, tal como entiendo el movimiento general de esta sección central del diálogo, es necesario abordar los argumentos que se ofrecen a continuación como un set. Con esto quiero decir que no debemos evaluar el alcance de cada argumento por separado, sino a partir de la consideración de su contribución específica a un movimiento dialéctico único, total y continuo. En este sentido, cada uno de los argumentos dialécticos ofrecidos arribaría, según la lectura propuesta, a soluciones parciales que, aun cuando en un principio puedan resultar medio difusas, en la estructura espiralada del diálogo van asumiendo progresivamente mayor nitidez y profundidad. En este sentido, el peso de cada argumento no debe evaluarse atómicamente y es por eso que, si asumimos una estructura trascendental del diálogo en general (más bien de toda la sección que comprende el análisis de la séptima definición del sofista), debemos percibir la contribución de cada argumento a la luz del movimiento argumentativo total.

Luego de exhibir las aporías a las cuales nos conduce el no ser, el Extranjero muestra que el ser nos pone en idéntica posición. La naturaleza dialéctica de la argumentación que emprenderá Platón a la hora de abordar la noción de ser e interpelar a quiénes se ocupan de "cuántos y cuáles son los entes" se manifiesta ya en el pasaje 243d, cuando el Extranjero afirma que "el método ( $\mu \varepsilon \dot{\varepsilon}$ oóov) consiste en hacer como si, estando ellos presentes, los interrogáramos así". Más allá del sentido más o menos técnico que porta aquí la noción de "método", la afirmación sitúa toda la discusión que se desplegará a continuación en un escenario nítidamente dialéctico. Inmediatamente el Extranjero cuestiona a los pluralistas y, a la hora de enfrentarlos, se sujeta al discurso que emiten para retrotraerse a sus condiciones. Así son interrogados:

Ustedes que dicen que todas las cosas son lo caliente, lo frío u alguna otra dupla de tal índole, ¿qué enuncian $(\varphi \theta \dot{\varepsilon} \gamma \gamma \varepsilon \sigma \theta \varepsilon)$ en realidad sobre ellos cuando dicen que ambos y cada uno es? ¿Qué hemos de suponer (i்o $\lambda \alpha \dot{\beta} \omega \mu \varepsilon v$ ) que es este 'ser'? ¿Es acaso una tercera cosa aparte de aquellas dos y entonces, según ustedes, hemos de sostener que el todo son tres cosas y no dos? Pues de alguna manera si llamaran 'ser' a uno u otro de los dos no

comentario al Crátilo (SSR v A 155), la cual traza idéntica identificación entre decir algo y decir algo que es.

${ }^{26} \mathrm{Al}$ respecto cfr. también el pasaje 240c: "El sofista de muchas cabezas nos ha obligado (†่vá $\left.\gamma \kappa \alpha \kappa \varepsilon v\right)$ a

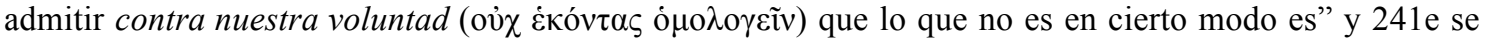
afirma que es en vano pretender hablar de falsedad sin caer en el ridículo "al ser forzado ( $\dot{\alpha} v \alpha \gamma \kappa \alpha \zeta o ́ \mu \varepsilon v o \varsigma)$ a contradecirse a sí mismo". 
estarían diciendo que ambos son igualmente: probablemente en ambos casos serían uno y no dos. (243d-e)

Y enseguida contempla otra posibilidad:

si intentaran llamar 'ser' a ambas cosas (...) aún así dirían claramente que aquellas dos son una. (243e-244a).

El Extranjero se sujeta así al discurso del adversario y se retrotrae a sus condiciones: hay que ir a aquello que está supuesto en la predicación del verbo "ser" respecto de los contrarios cuya existencia exclusiva se afirma. Entiendo que en el oscuro pasaje citado se ofrecen dos alternativas: o el ser es diferente de aquello de lo que se predica y en tal caso constituye una tercera cosa al nivel de las otras dos, de modo que admitiríamos la existencia de tres cosas y no de dos (por ejemplo, lo frío, lo cálido y el ser); o el ser se identifica con aquello de lo que se predica y en tal caso, tanto si partieran de la identificación de uno de los contrarios con el ser ("lo frío es" o "lo cálido es") como de ambos contrarios conjuntamente con el ser ("lo frío y lo cálido son"), estarían admitiendo que dos es uno, pues en el primer caso se admitiría sólo el ser de aquello que se identifica con el ser y en el segundo los contrarios se identificarían en y con el $\operatorname{ser}^{27} \mathrm{Al}$ margen de los problemas que entraña el argumento -fundamentalmente en lo que concierne al sentido que asume el verbo "ser"- se verifica que es la enunciación de su propia tesis lo que introduce al dualista en una aporía. ${ }^{28}$ Este argumento encontrará un claro eco en $254 \mathrm{~d}$, e involucra no solo las dificultades en torno al ser, sino también el problema de la predicación en general, tema que constituye el nudo del diálogo y será retomado más adelante al referirnos a la refutación que se ofrece frente a aquellos que niegan la posibilidad de decir una cosa a través de otra. Tal argumento permite no sólo rechazar la posibilidad de establecer un número preciso de cosas que son -pues se podrá aplicar idéntica estrategia a toda tesis que intente determinar cuantitativamente el ser-, sino también empezar a vislumbrar el vínculo y a la vez la distancia que guardan el ser y aquellas cosas de las cuales se predica. De suerte que la enorme problemática ínsita en la predicación ya se encontraría esbozada aquí.

El recurso al lenguaje y la predicación vuelve a exhibirse en la discusión con el monista que se ofrece a continuación. En el primer argumento (244b-d), el Extranjero refuta en dos tiempos a quienes afirman que todo es uno $(\ddot{\varepsilon} v \tau o ̀ ~ \pi \tilde{\alpha} v) .{ }^{29}$ En primer lugar les pregunta: "¿Dicen $(\varphi \alpha \tau \varepsilon)$ que solo hay algo uno?" poniendo de relieve la importancia de adscribir la enunciación del discurso al adversario. Y puesto que a continuación el monista reconoce que "llama ( $\alpha \lambda \lambda \varepsilon i \tau \varepsilon \varepsilon)$ 'ente' a algo", se concluye que utiliza dos nombres para lo mismo: "uno" y "ente". Esto, sin embargo, es inadmisible en la medida que se intente defender la tesis monista. Ante este cuestionamiento, señala el Extranjero, el interlocutor no podrá responder que constituyen

\footnotetext{
${ }^{27}$ En lo referente al elíptico pasaje que refiere a la posibilidad de identificar el ser con uno de los contrarios sigo la lectura de R. S. BLuCK, Plato's Sophist. A commentary, Manchester 1975, pp. 70-72 y CRIVelli, op. cit., pp. 73-74. Contra cfr. L. M. De RIJK, Plato's Sophist. A philosophical commentary, Amsterdam/Oxford/New York 1986, p. 95 y F. FronterotTA, Platone Sofista, Introduzione, traduzione e note, Milano 2007, pp. 346-348 n. 167. En lo concerniente a la tercera posibilidad, aquella que contempla que ambos se identifiquen con el ser, también está elidido el modo en que procede el argumento. Entiendo que idéntico argumento se encuentra en el tratado Sobre el no ser de Gorgias (DK82B3 76). En efecto, el segundo argumento para negar que tanto lo que es como lo que no es sean aquello que es, se apoya justamente en el hecho de que si ambos son, son lo mismo. Pero, sostiene el sofista, si son lo mismo (uno) no son ambos (dos), y si son ambos (dos) no son lo mismo (uno). Es posible percibir la matriz eleática deformada de Gorgias a lo largo de todo el Sofista, pero en casos como este señalar el paralelismo puede iluminar el sentido del texto.

${ }^{28}$ Que la discusión parte del lenguaje se ve corroborado por la profusa utilización que se hace de verbos de decir en estos pasajes. Los verbos $\varphi \eta \mu i ́, \lambda \hat{\varepsilon} \gamma \omega, \varphi \theta \dot{\varepsilon} \gamma \gamma \circ \mu \alpha \imath$ y $\kappa \alpha \lambda \dot{\varepsilon} \omega$ aparecen siete veces en este sintético argumento que va de $243 \mathrm{~d}$ a $244 \mathrm{~b}$.

${ }^{29}$ Aun cuando forma parte de la argumentación contra el monista, dejo de lado aquí el pasaje referido a la identidad entre ser y la totalidad (ő $\lambda \mathrm{ov}$ ) que va de $244 \mathrm{~d}$ a $245 \mathrm{e}$, porque no recurre a la enunciación de la tesis por parte del adversario, sino que se vale de un análisis conceptual de la noción de ö̉ov.
} 
dos nombres para lo mismo, puesto que él sostuvo que solo hay una cosa y en tal caso ya estaríamos frente a la presencia de dos nombres. El segundo momento del presente argumento va un paso más allá. En sentido estricto, señala el Extranjero, el monista tampoco podría admitir que se habla de la existencia de un nombre. Frente a la pregunta de si el nombre es diferente de

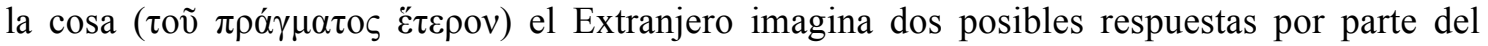
adversario, pero ambas conducen a aporía: si sostiene que el nombre es algo diferente (ह̌ $\tau \varepsilon \rho o v)$ de la cosa, debería admitir la existencia de dos cosas, el nombre y el ser. Si el nombre, en cambio, es lo mismo que la cosa, entonces caben dos posibilidades: (i) o el nombre no es nombre de nada (porque no hay nada diferente de él), lo cual parece entrañar un absurdo pues en tal caso el nombre dejaría de ser tal; (ii) o el nombre será sólo nombre del nombre, pero en ese caso también estaríamos frente a un desdoblamiento del nombre y la unidad debería ser, en este caso, unidad del nombre - por identificar este con el ser que se presumía uno-, razón por la cual tampoco se puede admitir tal posibilidad. ${ }^{30}$ Vemos pues que el argumento parte del discurso proferido por el monista y se retrotrae a sus condiciones, poniendo en primer plano la relación entre ser y discurso. Es el hecho de formular su propia tesis lo que en definitiva obliga al monista a admitir la pluralidad implicada en el discurso considerado tanto desde el punto de vista de la multiplicidad que supone en su interior, como desde el de la alteridad que guarda con relación a la referencia. Si intentamos delimitar la contribución de tal argumento al movimiento argumentativo total, se pone de relieve la postulación indirecta de la necesidad de la diferencia implicada en todo discurso, no solamente en el nivel de su estructura -cuestión que será desarrollada en $261 \mathrm{~d}-262 \mathrm{~d}$ al referirse al discurso como un combinación de nombre y verbo-, sino también a la de su relación con la referencia -punto supuesto en $262 \mathrm{~d}-263 \mathrm{~d}$, en que se definen verdad y falsedad-.

El último argumento constituye el nudo central del diálogo, desde mi punto de vista, y es el que, según entiendo, culmina en la mencionada afirmación de 259e: "el lógos se ha generado para nosotros por la combinación mutua de las formas". El análisis en torno al lenguaje tiene su origen en $251 \mathrm{a}-\mathrm{b}$, pasaje en el cual, frente a posiciones como la de los megáricos o los cínicos, Platón emprende la tarea de justificar por qué lo múltiple es uno y lo uno es múltiple. Este pasaje, muchas veces desatendido, es, sin embargo, un punto neurálgico del diálogo porque abre la discusión que culminará en la distinción de los cinco géneros mayores en tanto condición de cualquier tipo de discursividad. Afirma allí el Extranjero:

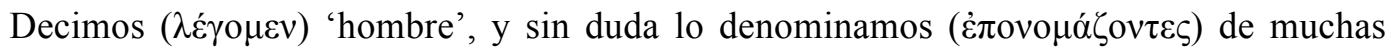

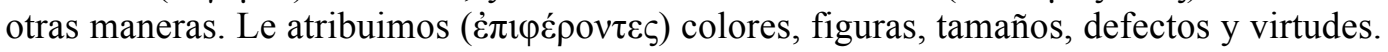
En todos aquellos casos y en muchos otros no sólo decimos ( $(\alpha \mu \varepsilon v)$ 'él es hombre', sino

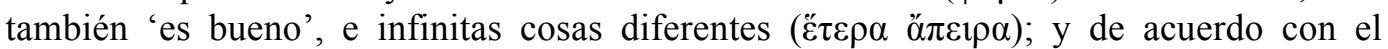
mismo argumento [procedemos] así respecto de las demás cosas: suponemos que cada una es una, y a su vez que la misma cosa es múltiple y con muchos nombres. (251a-b)

Y poco más adelante sostiene:

Cualquiera objetaría directa y fácilmente que es imposible que lo múltiple sea uno y lo uno sea múltiple, y sin duda, ellos se alegran al no permitir decir 'hombre bueno', sino "el bueno bueno", y "el hombre hombre". (251b-c)

El problema planteado aquí por Platón no parece ser meramente el de la atribución o predicación múltiple, que se haría patente allí cuando se asignan a una cosa múltiples características. El problema planteado involucra en rigor la predicación en general, incluyendo las atribuciones de ser y no ser. ${ }^{31}$ El lenguaje utilizado parece, sin embargo, enfocar a la

\footnotetext{
${ }^{30}$ Cfr. CRIVELli, op. cit., p. 74.

${ }^{31}$ El texto del ultimo pasaje citado no menciona la cópula y, según entiendo, en este caso es significativa la elisión, pues de acuerdo con lo afirmado en el contexto de la crítica al dualista en $243 \mathrm{~d}-\mathrm{e}$, el adversario considerará que atribuir ser a algo es atribuirle algo diferente de lo que él es. El mismo problema se
} 


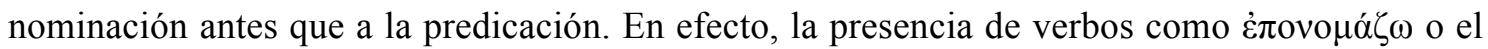
mismo sustantivo ővo $\mu \alpha$ en $251 \mathrm{a}-\mathrm{b}$ podrían conducirnos a pensar que lo que está en juego es la nominación. No es improbable que estos sean los términos en que el problema fue planteado por ciertos adversarios que Platón podría tener en mente -como ser el caso de Antístenes-, quienes habrían pensado el discurso bajo el modelo de la nominación. ${ }^{32}$ Frente a esta posición, Platón pone el foco en este diálogo en la predicación y establece la diferencia que guarda esta respecto del funcionamiento del nombre. Según surge de 261d-263d, Platón saldará este asunto estableciendo diferencias funcionales entre nombre y predicado: el nombre ancla el lógos a una referencia que por necesidad es; $y$, sobre la base de este anclaje y en función de la combinación con un predicado, el discurso podrá decir cosas que son o cosas que no son, dando lugar así a la posibilidad de la falsedad. De modo que el lenguaje de la nominación que aparece en el pasaje recién consignado debería atribuirse más bien a la concepción del interlocutor, que no establece diferencias entre nombrar y predicar. Esta es justamente una de las grandes empresas llevadas a término por Platón en este diálogo que, a la luz de la problemática de la predicación de ser respecto de movimiento y reposo, da lugar a la consideración que analizamos.

Más allá del destrato que recibe quien defiende la imposibilidad de la predicación por parte del Extranjero, ${ }^{33}$ es a partir de aquí que se introduce el problema central de la combinación en el enunciado y es en la indagación acerca de sus condiciones que el Extranjero recurrirá a la combinación de los géneros mayores. En efecto, la discusión se abre inmediatamente al plano ontológico cuando el Extranjero le plantea a Teeteto una alternativa que supone la distinción entre el plano ontológico y el plano del discurso y establece una relación entre ellos. Así, ofrece la siguiente alternativa: o nada se combina con nada -en tal caso, aclara, habría que establecerlo

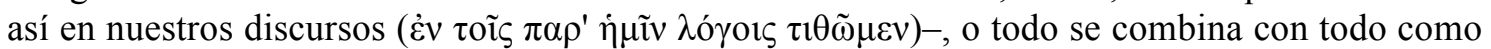
si fuese una sola cosa, o algunas cosas se combinan y otras no (251d-e).

Es muy importante reparar en el hecho de que aquí el Extranjero distingue dos planos en la discusión: para dar cuenta de la posibilidad de la predicación alude a una combinación entre cosas. De modo que ya no restringe su investigación al ámbito del discurso, sino que se remite al ámbito ontológico y pone en relación uno con otro, pues la famosa combinación de las formas (en este caso son mencionados tres de los géneros mayores, el ser, el movimiento y el reposo) viene justamente a ofrecerse como respuesta al problema de la predicación. ${ }^{34}$ Tal combinación opera justamente como condición de la combinación a nivel del discurso. Este paso del plano lógico al ontológico se sustenta, sin duda, en un principio no explicitado en el diálogo, según el cual el discurso guarda un cierto isomorfismo respecto del plano ontológico, que explicaría el movimiento de ida y vuelta que se realiza a lo largo de estos pasajes entre uno

evidencia en 249d-250d, pasaje que abre la problemática que culmina en el planteo que estamos analizando. Allí se establece que el hecho de sostener que movimiento y reposo son conduce a aporía. Estos no se identificaban con el ser, que constituye una tercera cosa diferente de uno y de otro. Sin embargo, señala el Extranjero, la alternativa movimiento-reposo es exhaustiva, esto es, no hay algo que no esté en movimiento o en reposo, a cuya luz resultaba conflictiva la afirmación del ser en tanto tercera cosa. Por eso el Extranjero concluye que el ser conduce a aporía al igual que el no ser. Más adelante establecerá que el ser puede atribuirse a cada una de las cosas que son, a pesar de $-\mathrm{o}$, más bien, en virtud de- no ser idéntico a ninguna de ellas. Al respecto cfr. también Teet. 201e-202a.

${ }^{32}$ Con respecto a este modo de concebir el discurso por parte de algunos sofistas cfr. I. CROMBIE, An Examination of Plato's Doctrines, vol. II, London 1963, pp. 477 y 482-484 y N. DENYER, Language, Thought and Falsehood in Ancient Greek Philosophy, London and New York 1993, pp. 12-14.

${ }^{33}$ Son "viejos instruidos tardíamente", "gente que por la pobreza de su intelecto" se sorprende frente a argumentos de esta índole, según el Extranjero (251c). En este sentido, el punto de partida parece análogo al asumido por Aristóteles en Metaph. Gamma 4: él también se refiere a gente que "por falta de educación" ( $\dot{\alpha} \pi \alpha 1 \delta \varepsilon v \sigma i ́ \alpha, 1006 \mathrm{a} 6)$ pide que cuestiones evidentes como el PNC sean demostradas. En uno y otro caso, sin embargo, las refutaciones representan la ocasión de ir a lo más profundo de sus respectivas ontologías para sentar sus bases últimas.

${ }^{34}$ Damos por supuesto aquí que formas y géneros son lo mismo en este diálogo. Con respecto a tal identidad y a los deslizamientos entre uno y otro término cfr. G. PECK, Plato's Sophist: the sumploké tôn eidôn, «Phronesis» VII (1962) pp. 46-66. 
y otro plano. ${ }^{35} \mathrm{Si}$, establece el Extranjero, frente a la pregunta formulada en relación con la posibilidad de la combinación entre los géneros, el adversario dijera que nada puede comunicarse con nada, el movimiento y el reposo no podrían participar del ser, "y en tal caso no podría afirmarse ni que el movimiento ni que el reposo son" (251e), pues esto supondría combinar uno u otro con el ser. Esto destruiría tanto a los defensores del movimiento como a los defensores de un ser inalterable, los condenaría directamente a la auto-refutación al verse en la necesidad de combinarlos en la propia enunciación de sus respectivas tesis. En rigor, si se rechaza toda combinación en el ámbito ontológico, no es posible discurso alguno. Así se verifica en el pasaje 252b, en que el Extranjero, refiriéndose ahora a quienes se han abordado el análisis del ser desde el punto de vista de la cantidad, establece:

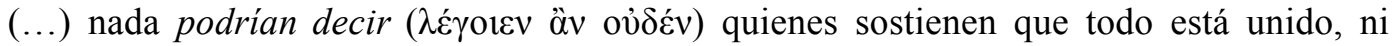

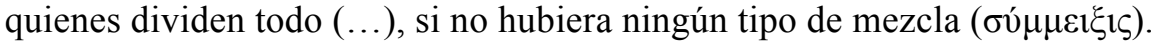

De suerte que sería el mismo recurso al lenguaje el que nos comprometería con la necesidad de la combinación en el plano ontológico. Así, el Extranjero se sirve de una argumentación radical también aquí al establecer que quienes niegan la combinación ontológica no podrán decir nada. La raíz trascendental de tal argumento se verifica con nitidez poco más adelante, cuando se refiere a quienes, por considerar que la combinación al nivel ontológico no es posible, niegan la legitimidad de la combinación al nivel del lógos:

Pero lo más ridículo de todo sería compartir el argumento de quienes no permiten enunciar una cosa a través de otra. (...) Respecto de todo se ven obligados a valerse de "ser" de "separado", de "lo otro" de "en sí" y de muchas otras expresiones que son

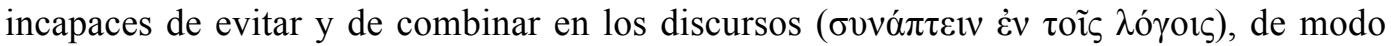
que no necesitan ser refutados: ellos, como suele decirse, tienen en su propia casa a su enemigo y a su contrincante y, como el insólito Euricles, ellos mismos llevan siempre de acá para allá una voz interior (252b8-c9).

De suerte que la prueba de Platón contra quienes niegan la combinación tanto a nivel ontológico como a nivel lógico es sencilla y bastante lapidaria: tales personajes radicalizados quedan relegados al silencio porque para enunciar sus propias teorías, necesitan poner al sujeto al cual se refieren en comunicación con el ser. Si no hubiera combinación, nada podrían decir. Los que se niegan a enunciar una cosa a través de otra no necesitan, pues, ser refutados, porque llevan la voz del adversario dentro como un ventrílocuo.

Una vez establecida la imposibilidad de que nada se combine con nada se examina la posibilidad de que todo se combine con todo. En tal caso, sostiene el Extranjero, deberíamos admitir la verdad de juicios como el que establece que el movimiento está en reposo o el reposo en movimiento, mas esto es absolutamente imposible (252d). Este argumento, a diferencia del anterior, no se apoya en la combinación al nivel del lenguaje, sino que emprende una analítica

\footnotetext{
${ }^{35}$ En este sentido, Platón no contempla la posibilidad de que la realidad no sea inteligible ni aprehensible por el discurso. Al respecto cfr. BALTZLY, op. cit., quien lleva al extremo el vínculo entre lenguaje y ser y a partir de un cruce con República, sostiene que los principios no hipotéticos a los que allí se refiere son aquellos cuya negación implicaría incurrir en auto-contradicción y que en última instancia es el bien el principio que garantiza tal optimismo al conferir ser e inteligibilidad a la realidad inteligible (p. 157). CASTAGNOLI argumenta consistentemente contra la tesis de BALTZLY y sostiene que debemos limitarnos a registrar el propósito que Platón atribuye explícitamente a la auto-refutación: silenciar a sus oponentes radicales y mostrar a cualquier otro cuán indeseable y ridícula es tal posición (op. cit., pp. 233-234). Según la posición que intentamos defender aquí, los propósitos no se limitan a los mencionados por CASTAGNOLI: aún cuando no sea posible establecer una relación tan estrecha como la que establece BALTZLY entre el método dialéctico de República y los argumentos examinados, es evidente que tal tipo de argumentación presenta una cara constructiva fundamental en el diálogo analizado. En este sentido, los argumentos no aspirarían meramente a exhibir la auto-contradicción implicada en la posición del adversario, sino que irían descubriendo ciertos principios fundamentales, lo cual nos habilita a considerarlos a la luz de las argumentaciones trascendentales.
} 
de los conceptos que conduce a establecer la necesaria falsedad de tales juicios. De modo que aquí se recurre tanto a la significación de los términos empleados como a la verdad de los juicios que los ligan. Descartadas las dos primeras posibilidades, queda entonces en pie la tercera opción: que algunas cosas se combinen y otras no. Como sucede en el caso de las letras, sostiene sugestivamente el Extranjero introduciendo así un modelo análogo de infinitas implicancias, habrá una gramática que establecerá los regímenes de combinaciones posibles y los que no. Quien quiera mostrar correctamente tales combinaciones entre géneros "deberá abrirse paso a través de los discursos ( $\delta i \grave{\alpha} \tau \tilde{\omega} v \lambda \hat{\gamma} \gamma \omega v)$ mediante una cierta ciencia $\left(\mu \varepsilon \tau^{\prime}\right.$ $\left.\dot{\varepsilon} \pi \iota \sigma \tau \eta \eta_{\zeta} \tau \imath v o ̀ \varsigma\right) "(253 \mathrm{~b})$. De suerte que el tránsito a través de los lógoi parece la vía de acceso al plano ontológico. ${ }^{36}$ Es competencia de la dialéctica -técnica ejercida por el filósofodistinguir "respecto de los géneros, cómo algunos son capaces de comunicarse con otros, y cómo no" (253e1-2). El análisis de tales combinaciones conduce entonces a una analítica que establece lo que podríamos llamar "patrones de predicación" legítimos. Partiendo de ciertos enunciados referidos a las nociones de reposo y movimiento, se infiere la necesidad de otros tres géneros: ser, identidad y diferencia, nociones que refieren a elementos constitutivos de todo lo real y cuya combinación mutua y con las cosas que son garantiza la posibilidad del lógos. La analítica conceptual de las nociones envueltas en tales enunciados redundará, pues, en la famosa combinación de las formas-géneros mayores. Así, sobre la base de ciertos esquemas de combinaciones posibles e imposibles, el Extranjero irá delineando una gramática ontológica que supondrá sacar a la luz la legalidad que rige las combinaciones entre los conceptos implicados. ${ }^{37}$ Una tarea tal conlleva una investigación de los diversos sentidos de 'ser', garantía de cualquier combinación en tanto forma relacional. ${ }^{38} \mathrm{Tal}$ combinación opera en última instancia como garantía última para otorgar legitimidad a la predicación, i.e. la combinación al nivel de los nombres, entendida en términos de atribución de cosas idénticas, en un caso, diferentes, en otro. En definitiva, si bien la sumploké de los géneros es independiente de las combinaciones que llevan a cabo los hombres en el discurso, ella les otorga, sin embargo, legitimidad y opera como condición de posibilidad de las diferentes operaciones que realizamos a través de la predicación. $^{39}$

\footnotetext{
${ }^{36} \mathrm{Cfr}$. también $254 \mathrm{c}$ en que se establece nuevamente la necesidad del recurso al lógos a la hora de examinar las combinaciones entre los géneros mayores. Resulta sugestivo el paralelo con el pasaje 99e del Fedón, en que Sócrates afirma la necesidad de refugiarse en los lógoi para acceder al conocimiento de lo real. Según entiendo, en ambos casos el lógos no sólo ofrece la vía para alcanzar el conocimiento, sino también "el lugar" en que la estructura de lo real se revela al hombre.

${ }^{37}$ Un antecedente de esta posición se encuentra en J. L. ACKRILL, SYMPLOKE EIDON in G. VLASTOS (ed.) Plato, A Collection of Critical Essays, New York 1971 (1955), pp. 199-206, quien fue el primero en establecer que entre el plano de las formas y el del lógos no hay una relación de representación, sino de presuposición. La combinación, según el autor, está presupuesta por todo enunciado y garantiza la significatividad, no la verdad de un enunciado. En última instancia, no se refiere más que a la compatibilidad e incompatibilidad de conceptos.

${ }^{38}$ No debe olvidarse que el puntapié inicial para toda la discusión sobre la combinación lo brindan las aporías que surgen a partir de la predicación de ser respecto del movimiento y el reposo. Entiendo que en última instancia identidad y diferencia garantizan la posibilidad de predicar ser sin identificar sujeto y predicado (no ser) e identificando ambos (identidad, movimiento de volver sobre sí). Acerca del ser en tanto forma relacional, N. L. CORDERO, La participation comme être de la forme dans le Sophiste de Platon, en N. L. Cordero (ed.) Ontologie et Dialogue, Paris 2000, pp. 33-46.

${ }^{39}$ Contra la línea iniciada por F. M. CORNFORD (Plato's Theory of Knowledge, London 1935, trad. esp. Barcelona 1983, pp. 271-286), considero que la combinación de las formas no viene a garantizar la verdad de los lógoi. En efecto, ya fueron establecidos hace tiempo los problemas que surgen de tal interpretación. Es claro que la verdad opera en el despliegue de las combinaciones en la medida que todo el análisis de 254b-256e supone, desde el punto de vista del Extranjero, el pasaje del enunciado a lo real. En este sentido, por necesidad la verdad de los enunciados concernientes a los géneros se ve comprometida. Pero de ahí no se sigue que la combinación de los formas sea condición del valor de verdad ni que dé cuenta del contenido de todo discurso. Considero que tampoco se ve directamente comprometida la significación de los discursos, punto que sí será abordado por el Extranjero en el análisis concerniente al lógos en tanto combinación de onómata. En los pasajes que se refieren a la combinación de los géneros, el tema no es mencionado siquiera por el Extranjero. Lo que se busca, según he intentado
} 
Entiendo que esta esquemática presentación del argumento para demostrar la combinación al nivel de los nombres es suficiente para percibir el sentido que asume el pasaje en que el Extranjero afirma que el lógos se ha generado para nosotros por la combinación mutua de las formas: tal combinación operará en última instancia como condición de todo discurso, modelo de todo discurso y causa misma del ser de todo discurso. ${ }^{40}$

A partir del somero examen ofrecido de las argumentaciones del Sofista, podemos sospechar que Aristóteles identificó esta estrategia de enorme eficacia a la hora de establecer principios y la retomó en el contexto de legitimar el primero de los principios de su propia ontología. ${ }^{41}$ En uno y otro caso, sin embargo, se verifican considerables diferencias. Hemos visto que Platón se vale en diversos sentidos del recurso al lenguaje: en la discusión librada con el dualista se sirve de la contradicción que se verifica en el contenido mismo del discurso del oponente, el cual establece la existencia de dos principios de lo real y, sin embargo, en la propia formulación de sus tesis se vale del ser como una tercera cosa; en la librada con el monista, en cambio, lo que involucra una contradicción es la existencia misma del discurso, que contradiría la tesis del monismo en tanto constituye una alteridad respecto del ser, hecho que el oponente no puede negar en la medida que se quiera enunciar su propia tesis; por último, en su enfrentamiento con el monismo lógico, aquel que impide decir una cosa a través de otra, el Extranjero se vale de la estructura misma del discurso en tanto articulación de la multiplicidad (lingüística y ontológica). Tal multiplicidad se exhibe en el propio discurso del oponente, de suerte que tal figura también recae en una contradicción al enunciar su propia tesis. Así, si bien los tres argumentos analizados del Sofista apuntan a diferentes planos comprometidos en el discurso, en todos ellos se verifica un recurso a la auto-refutación: el adversario sostiene una tesis cuya misma enunciación contradice. Aristóteles, en cambio, para no recaer en una petición de principio, no puede apuntar a la contradicción a la hora de refutar al negador del principio de no contradicción. Al adversario aristotélico no se le puede atribuir ninguna tesis en particular si es que todo es y no es a la vez y además, según hemos visto, resultaría cuestionable acusar de contradicción a un adversario que defiende la contradicción universal. Es por eso que, según hemos intentado mostrar, el negador del PNC es refutado en una instancia previa a le de la enunciación de la propia tesis: la de la significación. La argumentación aristotélica comprometería un plano previo entonces al de la enunciación.

Al margen de las diferencias señaladas entre las estrategias de las que se valen uno y otro filósofo, es claro que el estagirita retoma un modo de argumentación radical que le es funcional, al igual que a Platón, para asentar el carácter necesario de los principios en juego. Tal tipo de argumentación, según surge del análisis ofrecido, constituye un élenchos radical que acude no a las consecuencias sino más bien a las condiciones de un factum que es el lenguaje, instancia que el adversario no podrá rechazar si no quiere quedar excluido del escenario dialéctico. $^{42}$

exhibir iluminando algunos pasajes que, a mi entender, marcan el rumbo a seguir, es dar cuenta de la posibilidad de atribuir legítimamente ser y no ser, géneros implicados en toda predicación. En este sentido los mégista géne no constituyen cosas nombrables, sino que remiten a formas verbales básicas. Esto surge con nitidez a partir de la analogía empleada entre entre letras y formas. Al respecto cfr. G. RYLE, Letters and Syllables in Plato, «Philosophical Review» LVIX (1960) 4. pp. 431-451 y A. Soulez, La grammaire philosophique chez Platon, Paris 1991, pp. 113-146

${ }^{40}$ Respecto de esta triple causación de la combinación de las formas con relación al discurso cfr. F. FRONTEROTTA, op. cit. pp. 460-461, n. 259 e Introduzione 5.

${ }^{41}$ Este mecanismo de recurso a la dialéctica a la hora de establecer los principios de la ciencia puede identificarse también en Física I 2-3. Allí, para establecer la existencia del movimiento como principio de la física recurre a la refutación radical al monista, refutación que, por cierto, tiene importantes puntos de contacto no sólo con el referido argumento de Metafisica, sino también con la refutación al monismo ofrecida por Platón en Sofista.

${ }^{42}$ Agradezco la lectura atenta y las atinadas observaciones de Graciela Marcos, Lucas Álvarez y Marcelo Boeri de versiones previas del presente trabajo. Asimismo agradezco la generosidad de Beatriz Von Bilderling y Andrés Crelier, quienes me facilitaron material sobre argumentos trascendentales. 
CONICET (Argentina)

Universidad Nacional de Rosario

Universidad de Buenos Aires

pspangenberg@gmail.com 